\title{
The role of metformin hydrochloride in complex therapy of disorders of carbohydrate metabolism in patients with paranoid schizophrenia treated with atypical antipsychotics
}

\author{
Ivan Romash \\ Ivano-Frankivsk National Medical University, Ivano-Frankivsk, Ukraine
}

\begin{abstract}
Introduction. According to the literature, mortality among patients with schizophrenia is 1.5 -2 times higher than in the healthy population. One explanation for this is the complication of neuroleptic therapy, which, according to various authors, occurs in 2 to $100 \%$ of cases.

Purpose. We aimed to study some indicators of carbohydrate metabolism disorders in patients with paranoid schizophrenia who have been taking neuroleptics for a long time, to correct the established changes by adding mefformin hydrochloride to the standard regimen and to monitor its effectiveness.
\end{abstract}

Methodology. The study was conducted based on Municipal non-commercial enterprise "Precarpathian regional clinical center of mental health of Ivano-Frankivsk regional council. This study included patients diagnosed with paranoid schizophrenia according to the criteria of ICD10 (F20.0). As a result of our studies in 63 patients, we found a violation of carbohydrate metabolism, which accounted for 52\% of all examined. Among them, 55 patients with prediabetes: 12 (19.04\%) patients with impaired glucose tolerance (IGT), 43 (68\%) with impaired fasting glycemia (IFG), and 8 patients (12.7\%) with type 2 diabetes mellitus (T2D). Subsequently, all these 63 patients were prescribed corrective therapy with a drug from the group of biguanides - metformin hydroch/oride at a dose from 500 to 1000 mg/day: in violation of IFG at a dose of 500 mg/day; in case of IGT - 850 mg/day; in the case of T2D-1000 mg/day. All studies were performed before and after 3 months of metformin correction. These included fasting glucose, postprandial hyperglycemia (PPG) (two hours after a meal), glycosylated hemoglobin ( $H \mathrm{bAl} / \mathrm{C}$ ), immunoreactive insulin (IRI), and, if necessary, an oral glucose tolerance test (OGT). Fasting plasma glucose (FPG) was measured by the glucose oxidase method. HbAlc values were determined by ion-exchange high-performance liquid chromatography (HPLC). The determination of the IRI level was performed by enzyme-linked immunosorbent assay (ELISA)

Results and Discussion. The results of the research showed that $52 \%$ of all surveyed found disorders of carbohydrate metabolism. They were prescribed corrective therapy with a drug from the group of biguanides - metformin hydroch/oride at a dose of 500 to $1000 \mathrm{mg} / \mathrm{day}$. As a result of the research, we found that in all groups of examined patients revealed a positive dynamics of carbohydrate metabolism under the influence of this drug. A significantly higher therapeutic effect of the treatment of carbohydrate metabolism disorders with metformin was found in patients receiving the latter in combination with haloperidol. The combination of mefformin with risperidone and quetiapine showed a slightly lower clinical effect.

Conclusion. Our own clinical experience gives grounds to recommend mefformin hydrochloride as a medium for the correction of carbohydrate metabolism disorders in patients with a paranoid form of schizophrenia in the treatment of this category of patients with neuroleptics.

\section{Keywords}

carbohydrate metabolism, paranoid schizophrenia, atypical neuroleptics, metformin 
Address for correspondence:

Ivan Romash, M.D., Ph.D., assistant of professor Department of Psychiatry, Narcology and Medical Psychology Ivano-Frankivsk National Medical University, Ivano-Frankivsk, Ukraine, email: ivromash@ifnmu.edu.ua

This work is licensed under a Creative Commons Attribution-

NonCommercial 4.0 International License (CC BY-NC 4.0).

\section{(c) (i) (3)}

(C) Copyright: Romash, 2020

Licensee NDSAN (MFC- Coordinator of the NDSAN), Italy

DOI: hittp://dol.org/10.32437/mhgci.v3i1.93
Submitted for publication: 11 June 2020

Received: 11 June 2020

Accepted for publication: 17 October 2020

\section{Introduction}

According to the literature, mortality among patients with schizophrenia is 1.5 -2 times higher than in the healthy population $(1,2,3)$. One explanation for this is the complication of neuroleptic therapy, which, according to various authors, occurs in 2 to $100 \%$ of cases (IHME, 2018; Maruta et al., 2015).

Patients with schizophrenia, which is comorbid, need special attention somatic pathology, in particular metabolic disorders, which often go unnoticed by clinicians. Concomitant metabolic disorders in schizophrenia not only increase mortality but also create serious problems, in particular, in providing psychopharmacological care to this group of patients. According to scientific data, patients with paranoid schizophrenia comorbid with diabetes in $45 \%$ of cases in the first place put the treatment of somatic pathology, neglecting the treatment of the underlying disease, the other $40 \%$ - on the contrary - leave concomitant metabolic disorders without adequate correction (Jungsun, 2019; Romash, 2016, a).

The emergence of a new generation of antipsychotic pharmacotherapy, the so-called atypical antipsychotics (AA), largely devoid of the disadvantages of classical neuroleptics, has certainly been an important step forward in the treatment of patients with schizophrenia (Siskind et al., 2016). Several studies have confirmed the hypothesis of greater efficacy and safety of AA. Also, comparative studies have identified additional features of their clinical action: the ability to reduce secondary and possibly primary negative symptoms, reduce the severity of cognitive impairment, reduce comorbid affective symptoms, some drugs lack or low hyperprolactinemia and effectiveness in some cases, resistant to traditional neuroleptics (Siskind et al., 2018; Freyberg et.al., 2017; Romash, 2016).

However, several large recent studies have questioned the unconditional superiority of $A A$ over typical ones. This was due to the appearance of information about the presence of many metabolic side effects in AA, which lead to extremely serious consequences for physical health. According to the literature, the prevalence of metabolic syndrome (MS) in patients with schizophrenia is $37 \%-40 \%$, which is higher than in the general population (Siskind et al., 2018; IHME, 2018; Maruta et al., 2015; Romash, 2016).

Although "new" - atypical antipsychotic drugs cause fewer neurological side effects, they have a significant impact on the development of metabolic processes (Romash et al., 2016). In our recent studies were showed that neurological complications occur significantly more often on the background of taking a typical neuroleptic haloperidol than risperidone or quetiapine. In turn, a comparison of the presented AN showed that risperidone has a statistically higher probability of developing neurological complications compared to quetiapine. These data are consistent with recent studies by Spielmans G.I. et all., Oh G.H., who showed that of the currently known and widely used neuroleptics, risperidone has the highest level of akathisia, from $7 \%$ to $50 \%$, and the lowest incidence of akathisia is quetiapine (2 to $13 \%$ ). The incidence of akathisia in quetiapine is significantly lower than that of risperidone from $2 \%$ to $13 \%$. Complications from the functioning of the autonomic nervous system were also more common in patients of the haloperidol group. According to the data obtained, it should be noted that the use of atypical neuroleptics risperidone or quetiapine has a lower risk of developing late dyskinesia than with treatment with haloperidol. The study indicates the benefits of atypical antipsychotics mainly due to the lower severity of most neurological symptoms. Only some neurological symptoms in the examined patients were more common on the background of therapy with atypical antipsychotics. These results are consistent with the data of other authors who indicated a high probability of extrapyramidal side effects, including severe complications such as tardive dyskinesia, toxicoallergic reactions and neuroleptic malignant syndrome with haloperidol. Gardner M. D. and sang. Geddes J. R. et al. noted the development of tardive dyskinesia in patients treated with haloperidol for one year 17 times more often than with risperidone. However, according to $\mathrm{S}$. Leucht et al., the advantage of 
modern antipsychotics over drugs of previous generations is variable.

Therefore, in recent years, in addition to developing new drugs devoid of such side effects, more and more scientists from around the world have begun to look for rational concomitant corrective therapy. In particular, in their randomized 24-week double-blind, placebo-controlled study, Dan Siskind and coauthors studied the effect of concomitant metformin on weight change when clozapine was started. (Siskind et al., 2018) They demonstrated that co-initiation of metformin with the initiation of clozapine may reduce the burden of clozapine on cardiovascular and metabolic diseases.

\section{Purpose}

To investigate the effect of metformin corrective therapy on insulin resistance (IR) in patients with paranoid schizophrenia who had been taking neuroleptics for a long time.

\section{Methodology}

The study was conducted based on Municipal non-commercial enterprise "Precarpathian regional clinical center of mental health of IvanoFrankivsk regional council. This study included patients diagnosed with paranoid schizophrenia according to the criteria of ICD-10 (F20.0). The study was approved by the Bioethics Committee of Ivano-Frankivsk National Medical University and conducted following the principles of the Helsinki Declaration of the World Medical Association (Helsinki 1964, 2000 ed.). Before the study, all patients signed voluntary informed consent.

As a result of our studies in 63 patients, we found a violation of carbohydrate metabolism, which accounted for $52 \%$ of all examined. Among them, 55 patients with prediabetes: 12 (19.04\%) patients with impaired glucose tolerance (IGT), 43 (68\%) with impaired fasting glycemia (IFG), and 8 patients (12.7\%) with type 2 diabetes mellitus (T2D). Subsequently, all these 63 patients were prescribed corrective therapy with a drug from the group of biguanides - metformin hydrochloride at a dose from 500 to 1000 mg/day: in violation of IFG at a dose of 500 mg/day; in case of IGT - 850 mg/day; in the case of T2D- $1000 \mathrm{mg} /$ day. The initial dose in all groups was $500 \mathrm{mg}$ once daily with meals (breakfast or dinner), after 5-7 days, in the absence of gastrointestinal side effects, the dose was increased to $850-1000 \mathrm{mg}$ after breakfast or dinner. In case of side effects, the dose was reduced to the previous one and increased again after 5-7 days. Depending on the main 3- month therapy of paranoid schizophrenia preceding this stage of the study, patients were divided as follows: the first (I) Group included 15 patients receiving the typical neuroleptic haloperidol, the second (II) Group - 22 patients receiving atypical neuroleptic (AN) risperidone, to Group III - 15 patients who received atypical neuroleptic quetiapine. The fourth (IV) Group was a control group, which included 11 patients with paranoid schizophrenia in remission who did not receive neuroleptic therapy during the last 6 months. The duration of corrective therapy in patients of the study groups was 3 months.

It should be noted that the drug for concomitant corrective therapy was selected taking into account its mechanisms of action: reduces insulin resistance at the level of peripheral tissues (fat, muscle), increases glucose utilization by anaerobic glycolysis, slows glucose absorption in the intestinal tract, stops gluconeogenesis insulin in the liver and numerical benefits: low risk of hypoglycemia, promotes normalization and weight loss (anorexigenic effect), improves lipid profile, reduces the risk of developing type 2 diabetes in patients with impaired glucose tolerance, has a potential cardioprotective effect myocardial infarction in patients with obesity and type 2 diabetes; a small number of contraindications: hepatic insufficiency, GFR $<60 \mathrm{ml} / \mathrm{min}$., creatinine $>130$ $\mu \mathrm{mol} / \mathrm{l}$ in women and $120 \mu \mathrm{mol} / \mathrm{l}$ in men; and rare side effects: gastrointestinal phenomena. Also taken into account the experience of this drug by scientists such as Batista T., Henderson D. C. and Allison D. B. In addition, as proof of the safety of this drug is the fact that it can be used in children from 6 years. Therefore, it is important to mention the scientific study of Anagnostou E. et al. She used metformin hydrochloride to reduce weight in children with auricular disorders (aged 6 years) who were taking $A A$. In her study, metformin was more effective for weight loss with antipsychotics than placebo in this category of children (Anagnostou E. et al., 2016).

All studies were performed before and after 3 months of metformin correction. These included fasting glucose, postprandial hyperglycemia (PPG) (two hours after a meal), glycosylated hemoglobin (HbAlc), immunoreactive insulin (IRI), and, if necessary, an oral glucose tolerance test (OGTT). Fasting plasma glucose (FPG) was measured by the glucose oxidase method. $\mathrm{HbAlc}$ values were determined by ion-exchange high-performance liquid chromatography (HPLC). The determination of the IRI level was performed by enzyme-linked immunosorbent assay (ELISA).

We assessed carbohydrate metabolism according to the criteria of the International 
Diabetes Federation (IDF) -2005 classifications, and the metabolic syndrome was diagnosed according to the IDF-2007 criteria submitted by the working group of authors in the Adapted Clinical Regulation to the Unified Clinical Secondary Protocol care for type 2 diabetes. (Order of the Ministry of Health of Ukraine № 1118 of 21. 12. 2012. "On approval and implementation of medical and technological documents for the standardization of medical care for type 2 diabetes").

Statistical processing of the obtained results was performed using the program "STATISTICA 7.0." And the package of statistical functions of the program "Microsoft Excel, 2016". The reliability of the obtained results was confirmed based on the calculation of the Student's ratio. The arithmetic mean $(M)$ and its error $(m)$ were used to describe the quantitative features, the mean values were presented as $M \pm m$.

\section{Results and Discussion}

Corrective metformin therapy lasted 3 months. We evaluated the results of the initial and final data. Under the influence of corrective therapy, in all studied groups significantly decreased the rate of blood pressure (BP). Among men, this figure decreased by an average of $4.56 \%$, reaching an average of 93.81 $\mathrm{cm}$. Among women, BP decreased by $4.82 \%$, reaching $86.49 \mathrm{~cm}$.

Due to corrective therapy with metformin for 3 months, a significant decrease in body weight was found among patients of group I. Among the studied II and III groups there was a tendency to decrease body weight, but in comparison with the control group, it remains higher. We found a positive effect of biguanide therapy on body mass index (BMI): it decreased by $5.68 \%$ in Group I (haloperidol); by $3.79 \%$ - in Group II (risperidone) and by $2.29 \%$ - in Group III (quetiron). In groups II and III, BMl after 3 months of correction tended to decrease but remained probably higher compared to the Control group

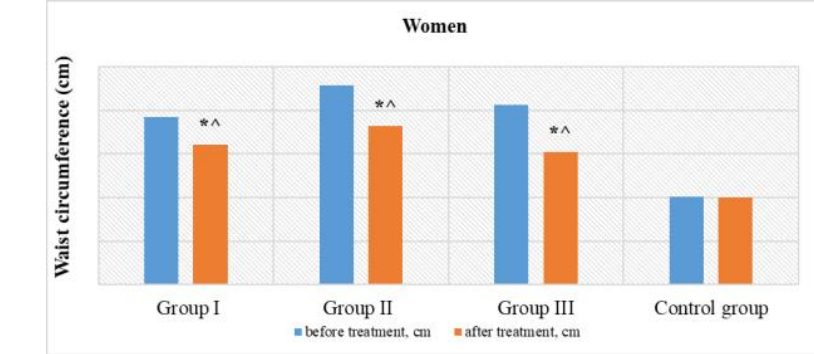

1)a

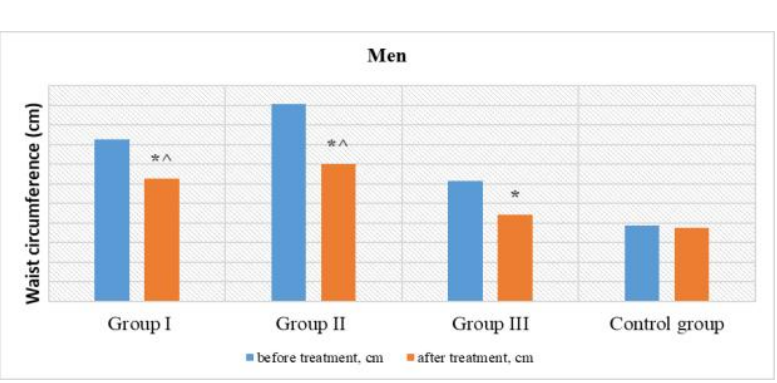

1)b

Fig. 1 a), 1b) Dynamics of changes in waist circumference under the influence of corrective therapy

Notes:

1. * - $(p<0.05)$ data are reliable for indicators before and after treatment.

2. $\wedge$ - $(p<0,05)$ data are reliable in comparison with the indices of the control group

As can be seen from the data in Fig. 1 a), 1b), in all groups studied significantly decreased waist circumference (WC). Among men, this figure decreased by an average of $4.56 \%$, reaching an average of $93.81 \mathrm{~cm}$. Among women, WC decreased by $4.82 \%$, reaching $86.49 \mathrm{~cm}$

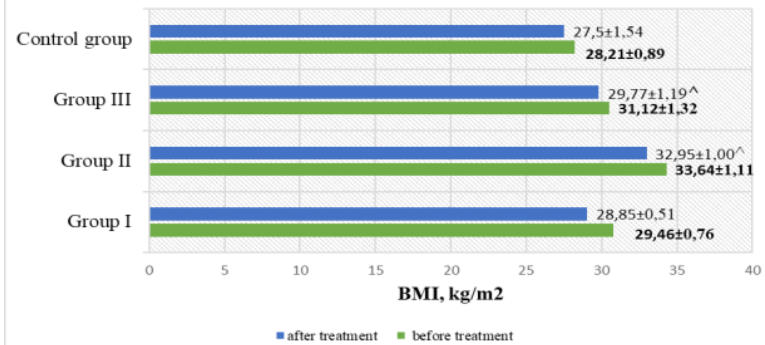

Fig.2 Dynamics of body weight in patients with paranoid schizophrenia before and after 3 months of correction with metformin hydrochloride.

Notes:

1. * - $(p<0.05)$ data are reliable for indicators before and after treatment.

2. $\wedge$ - $(p<0,05)$ data are reliable in comparison with the indices of the control group.

Due to corrective therapy with metformin for 3 months, we observe a probable decrease in body weight among patients of group I. Among the studied Groups II and III we see a tendency to decrease body weight, but in comparison with the control group, it is probably higher. We found a positive effect of biguanide therapy on BMl: this figure decreased by $5.68 \%$ in group I (haloperidol); by $3.79 \%$ - in Group II (risperidone) and by $2.29 \%$ - in Group III (quetiron). In Groups II and III, BMl after 3 months of correction tended to decrease but remained probably higher compared to the control group (Fig. 2). 
Consider the dynamics of carbohydrate metabolism in patients with paranoid schizophrenia who received corrective therapy with metformin. Fasting plasma glucose levels (Fig. 3) decreased by an average of $14.86 \%$, reaching an average of $6.23 \pm 1.76 \mathrm{mmol} / \mathrm{l}$ in all groups compared with the initial value of $7.40 \pm$ $0.26 \mathrm{mmol} / \mathrm{l}$, which is statistically significant.

In Group I, fasting blood glucose decreased from $7.01 \pm 0.29 \mathrm{mmol} / \mathrm{l}$ to $6.35 \pm 0.18 \mathrm{mmol} / \mathrm{l}$ after hypoglycemic therapy.

In Group II, glycemic parameters after corrective therapy had a significant decrease: from $8.44 \pm 0.23 \mathrm{mmol} / \mathrm{l}$ to $6.02 \pm 0.22 \mathrm{mmol} / \mathrm{l}$ $(p<0.05)$.

In Group III, the indicators also had a positive downward trend. Postprandial glycemia decreased by an average of $27.35 \%$, reaching an average of $7.03 \pm 0.38 \mathrm{mmo} / / \mathrm{liter}$

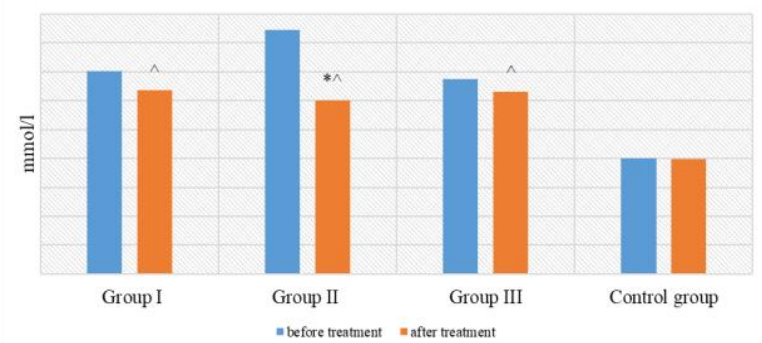

Fig. 3 Dynamics of fasting plasma glucose levels under the influence of corrective therapy with metformin.

Notes:

1. * - $(p<0.05)$ data are reliable for indicators before and after treatment.

2. ^ - $(p<0,05)$ data are reliable in relation to indicators in patients of the control group.

Statistially significant was the decrease of PPG in all study groups (Fig. 4)

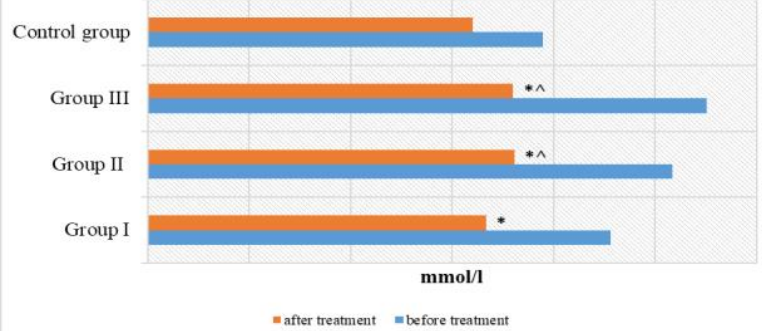

Fig. 4. Index of PPG in patients with paranoid schizophrenia before treatment and after 3 months of metformin correction.

Notes:

1. * - ( $p<0.05)$ data are reliable for indicators before and after treatment.

2. ^ - $(p<0,05)$ data are reliable in relation to indicators in patients of the control group.

The appointment of antidiabetic therapy had a positive effect on the prognostic value of
HbAlc. (Fig. 5.) This indicator decreased by an average of $16.01 \%$ : from $6.58 \pm 0.11 \%$ to 5.12 $\pm 0.12 \%$ in Group l; from $7.1 \pm 0.15 \%$ to $5.8 \pm$ $0.25 \%$ in Group II ( $p<0.05)$. In Group III there was also a tendency to reduce this indicator from $6.6 \pm 0.63 \%$ to $6.1 \pm 0.63 \%$.

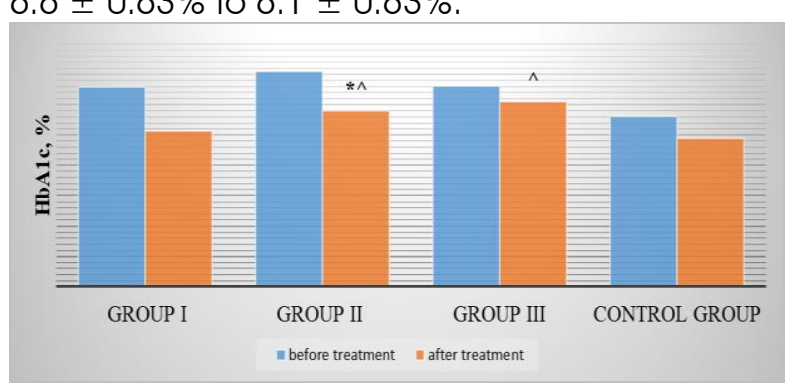

Fig. 5. Dynamics of glycosylated hemoglobin in patients with paranoid schizophrenia before treatment and after 3 months of metformin correction.

Notes:

1. * - $(p<0.05)$ data are reliable for indicators before and after treatment.

2. $\wedge$ - $(p<0,05)$ data are reliable in relation to indicators in patients of the control group.

No less important in the diagnostic value is the IRI indicator (Fig. 6). According to scientific data, this indicator is used to assess the degree of $\mathbb{I R}$ and functional activity of B-cells of the pancreas. In our case, IRI decreased by an average of $26.96 \%$ : achieving a significant decrease compared to the baseline of $25.56 \pm$ $0.70 \mu \mathrm{lU} / \mathrm{ml}$ to $13.40 \pm 0.35 \mu \mathrm{lU} / \mathrm{ml}$ in patients of Group I ( $\mathrm{p}<0,05)$; from $28.85 \pm 1.50 \mu \mathrm{lU} / \mathrm{ml}$ to $15.64 \pm 0.33 \mu \mathrm{lU} / \mathrm{ml}$ - Group II (p <0.05); from $26.49 \pm 0.69 \mu \mathrm{lU} / \mathrm{ml}$ to $14.56 \pm 0.46 \mu \mathrm{lU} / \mathrm{ml}$ Group III

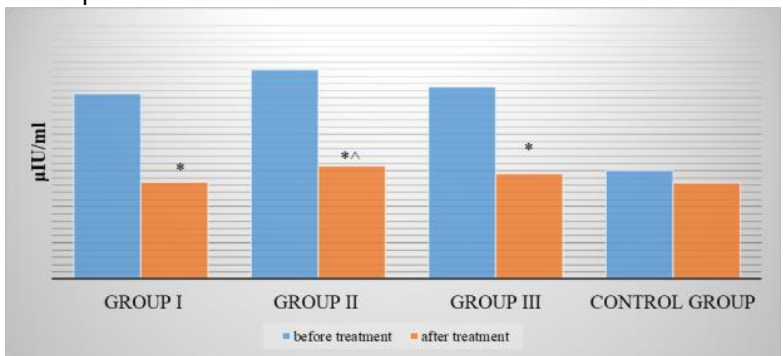

Fig. 6. Dynamics of IRI in patients with paranoid schizophrenia before treatment and after 3 months of metformin correction

Notes:

1. * - ( $\mathrm{p}<0.05)$ data are reliable for indicators before and after treatment.

2. $\wedge$ - $(p<0,05)$ data are reliable in relation to indicators in patients of the control group.

The value of the HOMA-IR index, which characterizes the $\mathbb{R}$, decreased by an average of $49.46 \%$ : from $7.96 \pm 0.75$ to $3.52 \pm 0.55$ in the group of patients taking haloperidol $(\mathrm{p}$ 
$<0.05)$; from $10.82 \pm 0.47$ to $5.97 \pm 0.5-$ risperidone $(\mathrm{p}<0.05)$; from $7.95 \pm 0.98$ to 4.15 \pm 0.98 - quetirone $(p<0.05)$

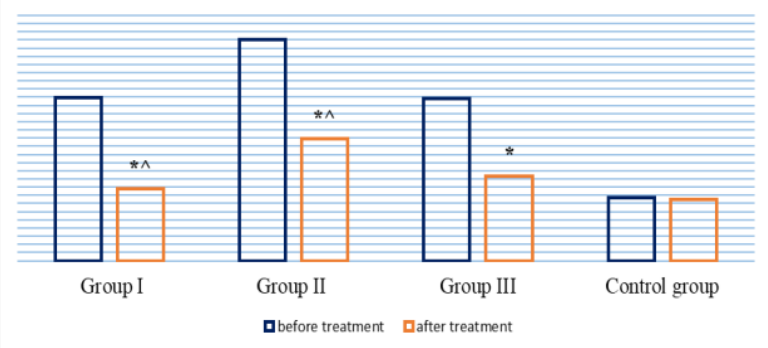

Fig. 7. Dynamics of the HOMA index in patients with paranoid schizophrenia before treatment and after 3 months of metformin correction

Notes:

1. * - ( $p<0.05)$ data are reliable for indicators before and after treatment.

2. ^ - $(\mathrm{p}<0,05)$ data are reliable in relation to indicators in patients of the control group.

Another, no less important, IP index - Caro. Under the influence of corrective therapy with metformin, this indicator increased in all three groups: in Group I by $74.04 \%$ reaching $0.47 \pm$ 0.02 ( $\mathrm{p}<0.05$ ); in Group II by $31.03 \%$ and amounted to $0.38 \pm 0.03$. In Group III, the Caro index probably increased by $72 \%$ reaching an average of $0.43 \pm 0.04(p<0.05)$

\section{Conclusion}

It was found that in all groups of examined patients revealed a positive dynamics of carbohydrate metabolism under the influence of metformin.

A significantly higher therapeutic effect of the treatment of carbohydrate metabolism disorders with metformin was found in patients receiving the latter in combination with haloperidol. The combination of metformin with risperidone and quetiapine showed a slightly lower clinical effect. Our own clinical experience gives grounds to recommend metformin hydrochloride as a medium for the correction of carbohydrate metabolism disorders in patients with a paranoid form of schizophrenia in the treatment of this category of patients with neuroleptics.

\section{Conflict of interest}

The Author declares that he has no conflict of interests

\section{References}

Anagnostou, E., Aman, M. G., Handen, B. L., Sanders, K. B., Shui, A., Hollway, J. A., Brian, J.,
Arnold, L. E., Capano, L., Hellings, J. A., Butter, E., Mankad, D., Tumuluru, R., Kettel, J., Newsom, C. R., Hadjiyannakis, S., Peleg, N., Odrobina, D., McAuliffe-Bellin, S., Zakroysky, P., ... Veenstra-VanderWeele, J. (2016). Metformin for Treatment of Overweight Induced by Atypical Antipsychotic Medication in Young People With Autism Spectrum Disorder: A Randomized Clinical Trial. JAMA psychiatry, 73(9), 928-937. https://doi.org/10.1001/jamapsychiatry.2016.1 232

Freyberg, Z., Aslanoglou, D., Shah, R., Ballon, J.S., 2017. Intrinsic and Antipsychotic Drug-Induced Metabolic Dysfunction in Schizophrenia. Frontiers

Neuroscience.https://doi.org/10.3389/fnins.201 7.00432

Institute for Health Metrics and Evaluation (IHME) (2018). Findings from the Global Burden of Disease Study 2017. Seattle, WA: IHME, 25 p.

International Diabetes Federation (IDF) (2015). Diabetes Atlas. 7th ed. Vancouver, Canada. Available at: https://www.idf.org/elibrary/epidemiology-research/diabetes-atlas

Jungsun, L. (2019). Metabolic Side Effects of Antipsychotic Medication: An Overview. J Korean Neuropsychiatr Assoc, 58(1):18-28. doi:10.4306/jknpa.2019.58.1.18

Maruta, N. O., Yaroslavtsev, S. O. (2015). Schizophrenia with comorbid mental and behavioral disorders (The structure of comorbid pathology, diagnostic criteria, prognostic factors and regularities of formation ). Medical psychology, 4: 46-50.

Order of the Ministry of Health of Ukraine dated 21.12.2012 No.1118 "On Approving and Implementation of Medical and Technical Documents on the Standardization of Medical Care in Type 2 Diabetes". (In Ukrainian). Available

at: https://zakon.rada.gov.ua/rada/show/v1 11828 2-12\#Text

Romash, I.R. (2016, a). Endogenous insulin and glucose concentration in patients with paranoid schizophrenia treated with atypical antipsychotics. The Pharma Innovation Journal, 5(4): 43-45. http://www.thepharmajournal.com/archives/20 16/vol5issue4/PartA/5-3-17.pdf

Romash, I.R. $(2016, b)$ Comparative analysis of neurological symptoms with stagnation of risperidone and haloperidol in patients with schizophrenia. Likars'ka sprava, 3-4:81-86.

Siskind, D. J., Leung, J., Russell, A. W., Wysoczanski, D., \& Kisely, S. (2016). Metformin for Clozapine Associated Obesity: A Systematic Review and Meta-Analysis. PloS 
one, $\quad 11(6), \quad e 0156208$. https://doi.org/10.1371/journal.pone.0156208 Siskind, D., Friend, N., Russell, A., McGrath, J. J., Lim, C., Patterson, S., Flaws, D., Stedman, T., Moudgil, V., Sardinha, S., Suetani, S., Kisely, S., Winckel, K., \& Baker, A. (2018). CoMET: a protocol for a randomised controlled trial of co-commencement of METformin as an adjunctive treatment to attenuate weight gain and metabolic syndrome in patients with schizophrenia newly commenced on clozapine. BMJ open, 8(3), e021000. https://doi.org/10.1 136/bmjopen-2017-021000 Oh, G.H., Yu, J.C., Choi, K.S. (2015) Simultaneous Comparison of Efficacy and Tolerability of Second-Generation Antipsichotics in Schizophrenia: Mixed-Treatment Comparison Analisis Based on Head-to Head Trial Data. $\begin{array}{lll}\text { Psychiatry Investig, } 12 & \text { (1):46-54 }\end{array}$ 